\title{
Complications of Bursectomy after Radical Gastrectomy for Gastric Cancer
}

\author{
Cuneyt Kayaalp $\cdot$ Turgut Piskin $\cdot$ Aydemir Olmez
}

Published online: 11 August 2011

(c) Société Internationale de Chirurgie 2011

\section{Dear Editor}

We were pleased to read the article by Imamura et al. [1] titled with "Influence of Bursectomy on Operative Morbidity and Mortality after Radical Gastrectomy for Gastric Cancer: Results of a Randomized Controlled Trial". In our center, we routinely perform bursectomy for radical gastrectomy for gastric cancers, and we agree that increased surgical experience can decrease the operative morbidity and mortality. However, we have some questions and comments about this article.

Although the authors found no difference of overall morbidity between bursectomy and nonbursectomy groups, we believe that the risk of increased specific complications that were representative for bursectomy should be clarified. As the authors mentioned, adhesions to the mesocolon and pancreas may cause specific local symptoms, such as delayed gastric emptying, afferent loop syndrome, or intestinal obstruction. There were five such complications in bursectomy group (4.8\%), and three of those patients required relaparotomy $(2.8 \%)$. There was only one $(1 \%)$ such complication in the nonbursectomy group and no related relaparotomy. Although the difference of those specific complications were not significant $(P=0.12)$, they were suggestive. What do the authors think about the effects of bursectomy on these specific complications?

In this series, the nonbursectomy group had a significantly greater number of combined organ resections. What were the complication rates of bursectomy and nonbursectomy groups when there were no combined organ resections? Although the authors reported that bursectomy required a longer operating time (median $27 \mathrm{~min}$ in patients with combined resection, $26 \mathrm{~min}$ in patients without a combined resection) the overall $P$ value in Table 2 was 0.368 . Was there any statistical difference or not between the groups for the operating time?

\section{References}

1. Imamura H, Kurokawa Y, Kawada J, Tsujinaka T, Takiguchi S, Fujiwara Y, Mori M, Doki Y (2011) Influence of bursectomy on operative morbidity and mortality after radical gastrectomy for gastric cancer: results of a randomized controlled trial. World J Surg 35:625-630. doi:10.1007/s00268-010-0914-5
C. Kayaalp $(\bowtie) \cdot T$. Piskin

Department of General Surgery, Inonu University,

Malatya, Turkey

e-mail: cuneytkayaalp@ hotmail.com

\section{A. Olmez}

Department of General Surgery, Yuzuncu Yil University,

Van, Turkey 